\title{
Sleep Medications and Women: a Review of Issues to Consider for Optimizing the Care of Women with Sleep Disorders
}

\author{
Andrew Krystal $^{1} \cdot$ Hrayr Attarian ${ }^{2}$
}

Published online: 26 October 2016

(C) Springer International Publishing AG 2016

\begin{abstract}
Certain medications exhibit sex-specific pharmacological profiles. It is, therefore, imperative to understand their unique pharmacokinetic properties to optimize women's health. Two such medications that are commonly used in sleep medicine are zolpidem and modafinil/armodafinil. The first is a sedative hypnotic while the latter, in both its incarnations, a wakepromoting agent. The slower clearance of these medications and higher serum concentrations after dosing in women are independent of weight and have to do with the impact of estrogen on the liver enzymes involved in the metabolism of these medications. Below, we summarize and discuss the data that is available on the sexspecific dosing of zolpidem, which in women, by FDA recommendations, is half of what is recommended in men. We also discuss the reasons behind the interaction of hormonal birth control and modafinil/armodafinil and rate of contraceptive failure when hormonal birth control is used with this wake-promoting agent.
\end{abstract}

Keywords Estrogen $\cdot$ Hormonal birth control $\cdot$ Zolpidem · Modafinil · Armodafinil CYP3A4

This article is part of the Topical Collection on Women and Sleep

Hrayr Attarian

h-attarian@northwestern.edu

1 Department of Psychiatry, Duke University Medical Center, Durham, NC, USA

2 Department of Neurology, Feinberg School of Medicine Northwestern University, Chicago, IL, USA

\section{Introduction}

To optimize clinical care of women with sleep disorders, it is critical to take into account the unique effects that some medications used to treat sleep disorders have on women. To illustrate this important aspect of gender-specific medicine, the review below highlights two medications commonly used to treat sleep disorders that have unique, sex-dependent pharmacological profiles. These are the insomnia medication zolpidem, and the wakefulness-promoting medications modafinil and its R-isomer armodafinil. Zolpidem is a benzodiazepine receptor agonist that binds to the GABA-A receptor [1]. It is one of the most commonly prescribed sedative hypnotics and is available in four formulations: immediate-release tablets $(5$ and $10 \mathrm{mg})$, controlled-release tablets $(6.25$ and $12.5 \mathrm{mg}$ ), sublingual dissolvable wafers (1.75 and $3.5 \mathrm{mg}$ ), and oral inhaler (5 mg per spray) [1,2]. Multiple trials have demonstrated zolpidem's efficacy in shortening sleep latency and improving subjective sleep, sleep efficiency, and continuity [1]. Most trials have demonstrated that its hypnotic effects are superior to placebo and some have shown equivalency to traditional benzodiazepines [1]. Zolpidem has demonstrated sustained efficacy over nightly use for a year without rebound or withdrawal symptoms upon cessation of treatment [3]. Zolpidem use has been associated with a number of adverse effects. These include sedation, dizziness, incoordination, nausea, headache, sleepwalking, sleep eating, and other complex behaviors, and it has abuse potential [4-8].

Modafinil and armodafinil are unique wake-promoting agents. Their specific mechanism of action remains unknown but a non-noradrenergic, dopamine-dependent adrenergic signaling mechanism has been proposed [9]. Both modafinil and armodafinil are indicated and proven effective in treating excessive daytime sleepiness in the setting of narcolepsy [10, $11]$, shift work disorder [12, 13], and obstructive sleep apnea 
$[11,14]$. Both drugs have lower abuse and dependence potential than the traditional stimulants [15] but cases of modafinil/ armodafinil dependence have been reported in the medical literature [16]. Their adverse event (AE) profile is favorable, and the only significantly related AEs include headache, nausea, anxiety or nervousness, insomnia, and dizziness [17].

In this article, we review the sex-specific issues associated with the pharmacological profiles of these drugs and discuss how consideration of these issues can play a role in optimizing clinical care.

\section{Sex Differences in Zolpidem Pharmacokinetics}

Of the medications used to treat insomnia, there is only one, zolpidem, where it has been reported that there are differential effects in men and women that are clinically important. Because this medication is the most commonly prescribed medication for the treatment of insomnia, this sex-based difference has the potential to have a significant impact on public health. It has been reported since 2000 that weight-normalized zolpidem clearance is lower in females compared with that in males 18. Since that time, this differential clearance has been demonstrated in terms of serum levels of zolpidem (pharmacokinetics) as well as in pharmacodynamic parameters including greater next-day driving impairment and the induction of sleep spindle activity in non-REM sleep [18-22]. The clinical implications of this difference are that for a given dose of zolpidem, women eliminate the drug more slowly and, as a result, have greater therapeutic and adverse effects than men. Because the effect is related to clearance of the molecule zolpidem from the blood, this sex-based difference is relevant for all formulations of zolpidem including the immediate-release version (original branded name Ambien in the USA), the controlled-release version (brand name Ambien $\mathrm{CR}$ ), the orally absorbed version (brand name Intermezzo), the intranasally administered version (brand name Zolpimist), and the orally dissolving formulation (brand name Edular).

The mechanism responsible for the sex difference in the pharmacokinetics of zolpidem has not been fully characterized. The key steps in the metabolism of zolpidem, which must be responsible for this difference, are as follows: hydroxylation of zolpidem by the cytochrome p450 3A4 isoenzyme (CYP3A4), oxidation by alcohol dehydrogenases, and conversion to a carboxylic acid by aldehyde dehydrogenases [23]. CYP3A4 is unlikely to be responsible for the observed sex difference in zolpidem pharmacokinetics as this isoenzyme has greater activity in females than that in males [23]. There is, however, some evidence that the sex difference in pharmacokinetics may be due to relatively lower expression of alcohol dehydrogenases and aldehyde dehydrogenases in women. This includes that androgens appear to affect the expression of alcohol dehydrogenases and aldehyde dehydrogenases resulting in lower gastrointestinal tract expression in women [23]. There is also evidence from a recent study in rats indicating that castration and the administration of an alcohol dehydrogenase and aldehyde dehydrogenase pathway inhibitor both led to comparable elevations in maximum concentration, time to maximum concentration, and area under the curve of serum level over time as seen in female rats [23].

Clinically, the relatively lower clearance of zolpidem in women should dictate that a lower zolpidem dosage should be used in women compared with that in men. Indeed, this is the recommendation of the U.S. FDA as communicated via a 2013 "Safety Announcement," which occurred in conjunction with changing the labeling to ensure that all forms of zolpidem had recommended dosages that were lower for women [24]. The relevant section of the "Safety Announcement" appears below:

FDA urges health care professionals to caution all patients (men and women) who use these zolpidem products about the risks of next-morning impairment for activities that require complete mental alertness, including driving. For zolpidem products, data show the risk for next-morning impairment is highest for patients taking the extended-release forms of these drugs (Ambien CR and generics). Women appear to be more susceptible to this risk because they eliminate zolpidem from their bodies more slowly than men.

Because use of lower doses of zolpidem will result in lower blood levels in the morning, FDA is requiring the manufacturers of Ambien, Ambien CR, Edluar, and Zolpimist to lower the recommended dose. FDA has informed the manufacturers that the recommended dose of zolpidem for women should be lowered from $10 \mathrm{mg}$ to $5 \mathrm{mg}$ for immediate-release products (Ambien, Edluar, and Zolpimist) and from $12.5 \mathrm{mg}$ to $6.25 \mathrm{mg}$ for extended-release products (Ambien CR). FDA also informed the manufacturers that, for men, the labeling should recommend that health care professionals consider prescribing the lower doses $-5 \mathrm{mg}$ for immediaterelease products and $6.25 \mathrm{mg}$ for extended-release products (see Dosing Recommendations).

The recommended doses of Intermezzo, a lower dose zolpidem product approved for middle-of-the-night awakenings, are not changing. At the time of Intermezzo's approval in November 2011, the label already recommended a lower dosage for women than for men.

Clinicians should take the sex-based differential clearance of zolpidem into account in their clinical practices to ensure the safety of women treated with zolpidem. This is best done by starting with the lowered FDA-recommended dosages in 
women and also warning women that even at these dosages, they may experience some next-day adverse effects. For the immediate-release version of zolpidem, if such next-day adverse effects occur, the clinician can recommend that the dosage can be further lowered by breaking the pill in half. This should not be done with other formulations of zolpidem. When adverse effects occur in women with the starting dose of these formulations, a switch to another agent is indicated.

\section{Sex Differences in the Pharmacokinetics of Modafinil and Armodafinil and the Effects of These Agents on Hormonal Birth Control}

Modafinil and armodafinil (the R-isomer of the racemic modafinil) are both metabolized hepatically through the cytochrome-P450 system (CYP) and are moderate inducers of the isoenzymes CYP2C10, CYP2B6, CYP1A2, and CYP3A4 $[25,26]$. Both drugs also have different pharmacokinetics in men vs. women. The mean value of peak plasma concentration $\left(\mathrm{C}_{\max }\right)$ is significantly higher in women than that in men 5.2 vs. $4.2 \mathrm{mg} / \mathrm{L}(p<0.05)$ after a single 200-mg dose of modafinil [27]. This difference remains valid ever after correction for body weight $(0.88$ vs. $0.72 \mathrm{ml} /$ $\mathrm{min} / \mathrm{kg}$ ) [25]. The clearance of the drugs is $30 \%$ higher in men than that in women, and plasma concentrations after a single dose are significantly higher in women than those in men [28]. The clearance of central compartment or rapid clearance (CL1) was $25.5 \%$ higher in men, intercompartmental clearance or slow clearance (CL2) was $35.4 \%$ higher, and peripheral volume distribution (V2) was $27.8 \%$ higher in men [28]. In a population pharmacokinetics trial, 50 healthy, young (mean age 22.4) men and women (M/F 1:1) were given a single 200-mg dose of modafinil. Participants continued fasting for $2 \mathrm{~h}$ after administration. The mean BMI of the study group was $21.9 \mathrm{~kg} / \mathrm{m}^{2}$. All women were in their luteal phase. During the test period, all the subjects were closely monitored and received a uniform diet. Blood samples were collected at $t=0,0.25,0.5$, $1,1.5,2,3,4,6,8,12,24,36$, and $48 \mathrm{~h}$ after the single modafinil dose [28]. Women also had higher plasma concentrations of modafinil than men, and the difference reached statistical significance with a $p<0.01$ [26]. Estrogen and other sex hormones also are metabolized through and interact with the CYP system [29]. Therefore, exogenous hormone intake may interact pharmacokinetically with modafinil or armodafinil. Hormonal contraception use among women is on the rise. According to the Guttmacher Institute, in 2012, $22 \%$ of women between the ages of 15 and 44 used a form of hormonal contraception. These include oral contraceptive pills, the vaginal rings, injectables, implants, and patches [30]. In 2013, the CDC reported that from 1982 to 2008, with the advent of alternatives to the pill (i.e., ring, patch, and implant), the proportion of hormonal contraceptive use has increased [31]. In 1982, $76.3 \%$ of sexually active women between the ages of 15 and 44 reported ever having used the pill while none reported using the ring, the patch, the injectables, or the implant primarily because they were not available then. In $2008,82.3 \%$ of the same demographics reported ever using the pill and $6.3 \%$ reported ever using the ring, $10 \%$ the patch, $22 \%$ the injectables, and $1.4 \%$ the implant [31].

Given the prevalence of hormonal contraception use, it is likely that most women of child-bearing age who will be started on modafinil or armodafinil will be using this particular method of birth control. The risk of reduced efficacy and increased clearance is not only theoretical based on the fact of common enzymatic pathway of metabolism. There is also practical data from human studies showing that the decrease of birth control efficacy is a real possibility with modafinil/ armodafinil.

In a placebo controlled single-blind study, 41 healthy female subjects on an oral contraceptive containing ethinyl estradiol and norgestimate were randomized either to modafinil (200 mg for 7 days and $400 \mathrm{mg}$ for 21 days) or placebo for 28 days. Those in the modafinil arm exhibited $18 \%$ decrease in ethinyl estradiol AUC $(0-\tau)$ and $11 \%$ decrease in ethinyl estradiol $\mathrm{C}_{\max }[32]$.

In a large survey of general practitioners (GP) in the UK, 599 girls and women were identified as having been placed on modafinil for a variety of indications. Out of these, 223 were of child-bearing age and 97 were using hormonal contraception. Three of these 97 reported unplanned pregnancies while compliantly taking hormonal contraception [33].

As a result of these two studies, both modafinil and armodafinil include a warning in their package inserts about the potential interaction between these two medications and hormonal birth control.

Since both modafinil and armodafinil are pregnancy category $\mathrm{C}$, it is important to prevent unexpected pregnancies in patients on modafinil and armodafinil. Women tend to have higher concentrations of these medications as well as slowed clearance [34]. This allows for higher chance of interaction with hormonal contraception. Therefore, it is imperative to increase awareness among clinicians prescribing these drugs of this interaction, and it is equally essential for them to advise their patients of the potential failure of hormonal contraception.

Practitioners prescribing modafinil or armodafinil to women on hormonal contraception should advise their patients of the need to use alternate or supplemental methods of birth control. One alternative is to switch from their contraceptive method to an intrauterine device or an IUD. Another is to use an additional barrier method such as a diaphragm or a condom. By avoiding hormonal contraception, women on armodafinil or modafinil will avoid the risk of accidental pregnancy. 


\section{Discussion and Conclusions}

The above discussions of zolpidem and modafinil/armodafinil illustrate the need for a sex-specific mindset while pharmacologically treating sleep disorders in adults. Even while treating sleep disorders that are not due to hormonal changes or life circumstances specific to women (such as pregnancy and menopause), sex and gender can play a major role in patients' response to treatment. Because of differential metabolism of zolpidem, it is essential that women be started on the lower dose of whichever formulation is chosen. These include $5 \mathrm{mg}$ of zolpidem, $6.25 \mathrm{mg}$ of zolpidem CR, and $1.75 \mathrm{mg}$ of sublingual zolpidem. To those women of child-bearing age taking a form of hormonal contraception other than an IUD who need modafinil/armodafinil for OSA-related residual fatigue, shift work disorder, or a primary hypersomnia [35], and sometimes for the treatment of MS related fatigue [36] a thorough education on the interaction between these drugs and their hormonal birth control must be provided. In addition, it should be recommended that they use a barrier method or switch to an IUD or other non-hormonal contraceptive modalities. Zolpidem and modafinil/armodafinil also illustrate the importance of characterizing sex differences in the pharmacokinetics and pharmacodynamics of all medications and incorporating these characterizations into practice. Zolpidem is a particularly notable case of a lag in incorporation into practice. Data on the sex difference in pharmacokinetics were first published in 2000. However, it was not until the 2013 FDA "Safety Announcement" that this difference attracted the widespread attention of practitioners and was applied in the routine treatment of patients. Hopefully, with systematic collection and incorporation of data on sex differences, we can eliminate such time lags with newly emerging and future medications.

Acknowledgments The authors would like to acknowledge the Society of Women's Health Research for providing this opportunity to write this very timely review.

\section{Compliance with Ethical Standards}

Conflict of Interest Andrew Krystal and Hrayr Attarian declare that they have no conflict of interest.

Human and Animal Rights and Informed Consent This article does not contain any studies with human or animal subjects performed by any of the authors.

\section{References}

1. MacFarlane J, Morin CM, Montplaisir J. Hypnotics in insomnia: the experience of zolpidem. Clin Ther. 2014;36(11):1676-701.

2. Neubauer DN. ZolpiMist: a new formulation of zolpidem tartrate for the short-term treatment of insomnia in the US. Nat Sci Sleep. 2010;2:79-84.
3. Roehrs TA et al. Twelve months of nightly zolpidem does not lead to rebound insomnia or withdrawal symptoms: a prospective placebo-controlled study. J Psychopharmacol. 2012;26(8):108895.

4. Poceta JS. Zolpidem ingestion, automatisms, and sleep driving: a clinical and legal case series. J Clin Sleep Med. 2011;7(6):632-8.

5. Licata $\mathrm{SC}$ et al. Modest abuse-related subjective effects of zolpidem in drug-naive volunteers. Behav Pharmacol. 2011;22(2):160-6.

6. Hwang TJ et al. Risk predictors for hypnosedative-related complex sleep behaviors: a retrospective, cross-sectional pilot study. J Clin Psychiatry. 2010;71(10):1331-5.

7. Scharf MB, Roth T, Vogel GW, Walsh JK. A multicenter, placebocontrolled study evaluating zolpidem in the treatment of chronic insomnia. J Clin Psychiatry. 1994;55(5):192-9.

8. Roth T, Roehrs T, Vogel G. Zolpidem in the treatment of transient insomnia: a double-blind, randomized comparison with placebo. Sleep. 1995;18(4):246-51.

9. Wisor JP, Eriksson KS. Dopaminergic-adrenergic interactions in the wake promoting mechanism of modafinil. Neuroscience. 2005;132(4):1027-34.

10. US Modafinil in Narcolepsy Multicenter Study Group. Randomized trial of modafinil for the treatment of pathological somnolence in narcolepsy. US Modafinil in Narcolepsy Multicenter Study Group. Ann Neurol. 1998;43(1):88-97.

11. Black JE et al. The long-term tolerability and efficacy of armodafinil in patients with excessive sleepiness associated with treated obstructive sleep apnea, shift work disorder, or narcolepsy: an open-label extension study. J Clin Sleep Med. 2010;6(5):45866.

12. Darwish M, Bond M, Ezzet F. Armodafinil and modafinil in patients with excessive sleepiness associated with shift work disorder: a pharmacokinetic/pharmacodynamic model for predicting and comparing their concentration-effect relationships. J Clin Pharmacol. 2012;52(9):1328-42.

13. Czeisler CA et al. Modafinil for excessive sleepiness associated with shift-work sleep disorder. N Engl J Med. 2005;353(5):476-86.

14. Inoue Y, Takasaki Y, Yamashiro Y. Efficacy and safety of adjunctive modafinil treatment on residual excessive daytime sleepiness among nasal continuous positive airway pressure-treated japanese patients with obstructive sleep apnea syndrome: a double-blind placebo-controlled study. J Clin Sleep Med. 2013;9(8):751-7.

15. Morgan PT et al. Modafinil and sleep architecture in an inpatientoutpatient treatment study of cocaine dependence. Drug Alcohol Depend. 2016;160:49-56.

16. Krishnan R, Chary KV. A rare case modafinil dependence. J Pharmacol Pharmacother. 2015;6(1):49-50.

17. Kuan YC et al. Effects of modafinil and armodafinil in patients with obstructive sleep apnea: a meta-analysis of randomized controlled trials. Clin Ther. 2016;38(4):874-88.

18. Greenblatt DJ et al. Comparative kinetics and response to the benzodiazepine agonists triazolam and zolpidem: evaluation of sexdependent differences. J Pharmacol Exp Ther. 2000;293(2):43543.

19. Dijk DJ et al. Sex differences and the effect of gaboxadol and zolpidem on EEG power spectra in NREM and REM sleep. $J$ Psychopharmacol. 2010;24(11):1613-8.

20. Greenblatt DJ et al. Gender differences in pharmacokinetics and pharmacodynamics of zolpidem following sublingual administration. J Clin Pharmacol. 2014;54(3):282-90.

21. Olubodun JO et al. Pharmacokinetic properties of zolpidem in elderly and young adults: possible modulation by testosterone in men. Br J Clin Pharmacol. 2003;56(3):297-304.

22. Verster JC, Roth T. Gender differences in highway driving performance after administration of sleep medication: a review of the literature. Traffic Inj Prev. 2012;13(3):286-92. 
23. Peer CJ, Strope JD, Beedie S, Ley AM, Holly A, Calis K, et al. Alcohol and aldehyde dehydrogenases contribute to sex-related differences in clearance of zolpidem in rats. Front Pharmacol. 2016;7: 260. doi:10.3389/fphar.2016.00260. eCollection 2016.

24. FDA. FDA drug safety communication: risk of next-morning impairment after use of insomnia drugs; FDA requires lower recommended doses for certain drugs containing zolpidem (Ambien, Ambien CR, Edluar, and Zolpimist) 2013.

25. Robertson Jr P, Hellriegel ET. Clinical pharmacokinetic profile of modafinil. Clin Pharmacokinet. 2003;42(2):123-37.

26. Darwish $\mathrm{M}$ et al. Evaluation of the potential for a pharmacokinetic drug-drug interaction between armodafinil and ziprasidone in healthy adults. Clin Drug Investig. 2014;34(10):691-9.

27. Goss AJ et al. Modafinil augmentation therapy in unipolar and bipolar depression: a systematic review and meta-analysis of randomized controlled trials. J Clin Psychiatry. 2013;74(11):1101-7.

28. Tao $\mathrm{G}$ et al. Population pharmacokinetics of modafinil in Chinese Han, Mongolian, Korean, Uygur, and Hui healthy subjects determined by nonlinear mixed-effects modeling. Ther Drug Monit. 2010;32(2):189-93.

29. Somner J et al. Polymorphisms in the P450 c17 (17-hydroxylase/ 17,20-Lyase) and P450 c19 (aromatase) genes: association with serum sex steroid concentrations and bone mineral density in postmenopausal women. J Clin Endocrinol Metab. 2004;89(1):344-51.
30. Unknown. Guttmacher Institute fact sheet: contraceptive use in the United States. 2015 October 2015 [cited 2016 June 7th].

31. Mosher W, Jones J. Use of contraception in the United States: 1982-2008. Vital Health Stat. 2010;23(29):1-37.

32. Robertson Jr P et al. Effect of modafinil on the pharmacokinetics of ethinyl estradiol and triazolam in healthy volunteers. Clin Pharmacol Ther. 2002;71(1):46-56.

33. Davies M, Wilton L, Shakir S. Safety profile of modafinil across a range of prescribing indications, including off-label use, in a primary care setting in England: results of a modified prescription-event monitoring study. Drug Saf. 2013;36(4):237-46.

34. Darwish $\mathrm{M}$ et al. Armodafinil and modafinil have substantially different pharmacokinetic profiles despite having the same terminal half-lives: analysis of data from three randomized, single-dose, pharmacokinetic studies. Clin Drug Investig. 2009;29(9):613-23.

35. Mayer $\mathrm{G}$ et al. Modafinil in the treatment of idiopathic hypersomnia without long sleep time - a randomized, double-blind, placebocontrolled study. J Sleep Res. 2015;24(1):74-81.

36. Asano M, Finlayson ML. Meta-analysis of three different types of fatigue management interventions for people with multiple sclerosis: exercise, education, and medication. Mult Scler Int. 2014;2014: 798285 . 